\title{
Recovery of an Injured Corticoreticulospinal Tract in a Patient With Cerebral Infarct
}

\author{
Sungho Jang, MD, Jeongpyo Seo, PhD, Soyoung Kwak, MD, MPH, PhD
}

Department of Physical Medicine and Rehabilitation, Yeungnam University College of Medicine, Daegu, Korea

A 66-year-old right-handed female patient developed absolute weakness of the right lower extremity (Manual Muscle Test [MMT], 0/5), following an infarct in the left supplementary motor area and premotor cortex (Fig. 1A). She underwent conservative management at the neurology department of a university hospital. Two weeks after the infarct, she was transferred to the rehabilitation department of the same university hospital. The weakness of the right lower extremity was similar to that seen at the time of the infarct (MMT, 0/5). She underwent comprehensive rehabilitative management, including neurotropic drugs (pramipexole, amantadine, and venlafaxine), movement therapy, and neuromuscular electrical stimulation of the right knee extensor and ankle dorsiflexor muscles. After rehabilitation for 6 weeks, there was significant motor recovery of her right lower extremity (MMT: hip flexor, 3; knee extensor, 3; and ankle dorsiflexor, 0). At 8 weeks after the onset, she was able to walk independently on an even floor, fitted with an ankle-footorthosis.

Diffusion tensor imaging (DTI) data were acquired twice (at 2 and 8 weeks after the onset). DTI was performed using a sensitivity-encoding head coil on a 1.5-T. For analysis of the corticospinal tract (CST), the first and second regions of interest (ROIs) were placed at the anterior blue portion of the upper and lower pons, respectively, on the axial image of a color map. For reconstruction of the corticoreticulospinal tract (CRT), the first ROI was placed on the reticular formation of the medulla, and the second ROI was placed on the tegmentum of the midbrain. At the 2-week diffusion tensor tractography (DTT), the integrity of both the CSTs and the right CRT of the patient were preserved. However, the left CRT showed discontinuation at the brainstem level (Fig. 1B). By contrast, the 8-week DTT revealed the discontinued left CRT was connected to the left cerebral cortex (the changes of the DTT parameters are described in Supplementary Table S1).

The 2-week DTT showed that the left CRT was discontinued at the brainstem level, suggesting severe injury of the left CRT, which may have degenerated following injury due to the left cerebral infarct. Elongation of the discontinued left CRT to the cerebral cortex on 8-week DTT appears to indicate a recovery of the injured left CRT. We believe this recovery of the left CRT is partly attributed to the motor recovery of the right lower extremity in this patient. In conclusion, using DTT, we hereby demonstrate the recovery of an injured CRT in the affected hemisphere of a patient with a cerebral infarct, concurrent with the motor recovery of the affected lower extremity [1-4].

Received March 8, 2017; Accepted April 3, 2017

Corresponding author: Soyoung Kwak

Department of Physical Medicine and Rehabilitation, Yeungnam University College of Medicine, 170 Hyeonchung-ro, Nam-gu, Daegu 42415, Korea. Tel: +82-53-620-4098, Fax: +82-53-620-4098, E-mail: soyoung.kwak@daum.net

ORCID: Sungho Jang (http://orcid.org/0000-0001-6383-5505); Jeongpyo Seo (http://orcid.org/0000-0002-2695-7957); Soyoung Kwak (http://orcid. org/0000-0002-1031-7197).

(c) This is an open-access article distributed under the terms of the Creative Commons Attribution Non-Commercial License (http://creativecommons.org/ licenses/by-nc/4.0) which permits unrestricted noncommercial use, distribution, and reproduction in any medium, provided the original work is properly cited. Copyright $\odot 2017$ by Korean Academy of Rehabilitation Medicine 
(A)

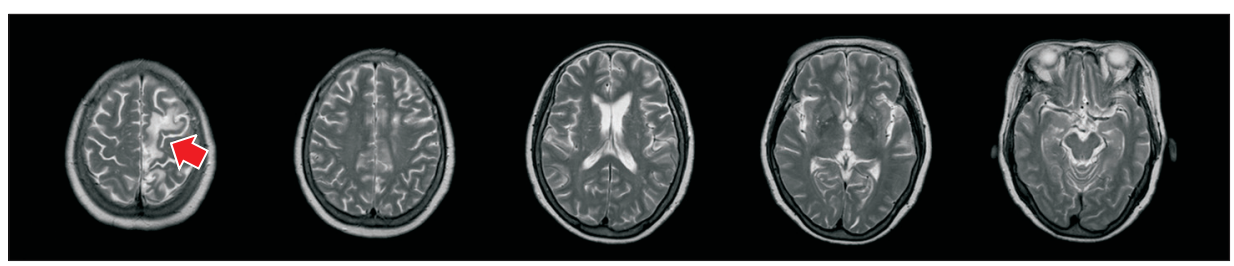

(B)

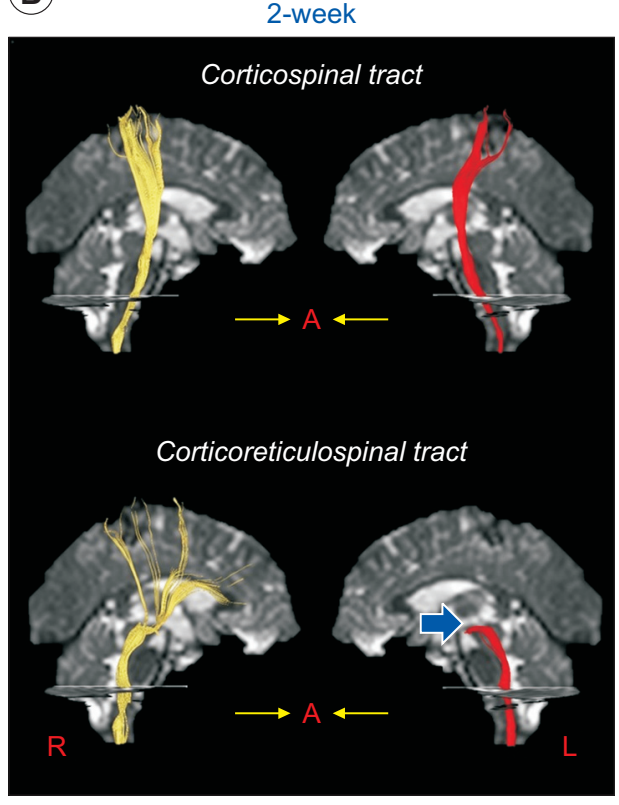

8-week

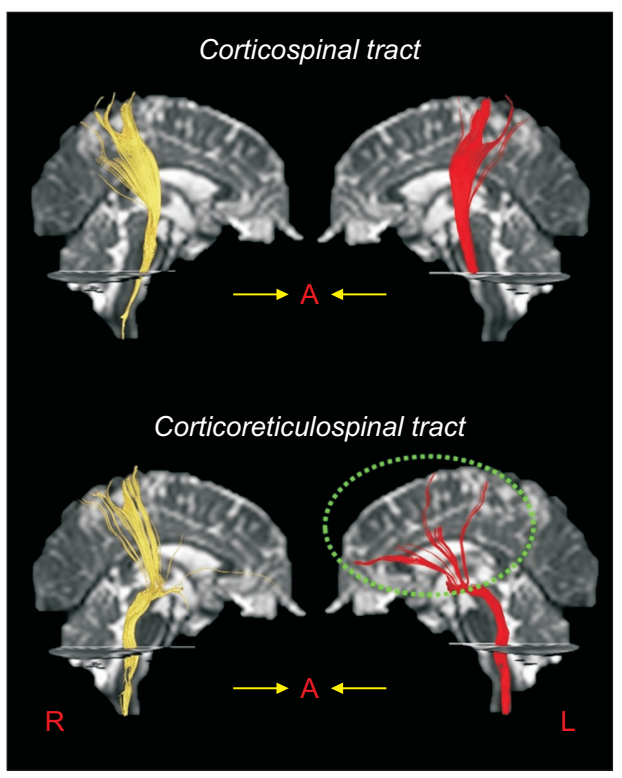

Fig. 1. (A) Brain MR images at 2 weeks after onset shows leukomalactic lesion (red arrow) in the left supplementary motor area and premotor cortex. (B) Results of diffusion tensor tractography (DTT) for the corticospinal tract (CST) and the corticoreticulospinal tract (CRT) of the patient. The 2-week DTT shows the integrity of both the CSTs and the right CRT of the patient are preserved. However, the left CRT shows discontinuation (blue arrow) at the brainstem level. By contrast, the 8-week DTT reveals the discontinued left CRT is connected to the left cerebral cortex (dotted circle).

\section{CONFLICT OF INTEREST}

No potential conflict of interest relevant to this article was reported.

\section{ACKNOWLEDGMENTS}

This work was supported by the National Research Foundation (NRF) of Korea grant funded by the Korean Government (MSIP) (No. 2015R1A2A2A01004073).

\section{SUPPLEMENTARY MATERIALS}

Supplementary materials can be found via http://doi. org/10.5535/arm.2017.41.3.516. Table S1. DTT parameters of corticoreticulospinal tract and corticospinal tract of the patients at 2 weeks and 8 weeks after the onset of stroke.

\section{REFERENCES}

1. Yeo SS, Jang SH. Recovery of an injured corticospinal tract and an injured corticoreticular pathway in a patient with intracerebral hemorrhage. NeuroRehabilitation 2013;32:305-9.

2. Jang SH, Yeo SS. Recovery of an injured corticoreticular pathway via transcallosal fibers in a patient with intracerebral hemorrhage. BMC Neurol 2014;14:108.

3. Jang SH, Lee J, Lee HD. Peri-infarct reorganization of an injured corticoreticulospinal tract in a patient with cerebral infarct. Int J Stroke 2015;10:E62-3.

4. Jang SH, Lee HD. Recovery of the Corticoreticulospinal Tract Injured by a Subfalcine Herniation in a Patient with Traumatic Brain Injury. Am J Phys Med Rehabil 2016;95:e60-1. 


\section{SUPPLEMENTARY MATERIALS}

Table S1. DTT parameters of corticoreticulospinal tract and corticospinal tract of the patients at 2 weeks and 8 weeks after the onset of stroke

\begin{tabular}{|c|c|c|c|c|}
\hline & & & 2-week DTT & 8-week DTT \\
\hline \multirow[t]{6}{*}{ Affected hemisphere } & Lt. CRT & Fiber number & 429 & 1,116 \\
\hline & & FA & 0.430 & 0.468 \\
\hline & & $\mathrm{ADC}$ & 0.948 & 0.923 \\
\hline & Lt. CST & Fiber number & 876 & 999 \\
\hline & & FA & 0.505 & 0.537 \\
\hline & & $\mathrm{ADC}$ & 0.999 & 0.953 \\
\hline \multirow[t]{6}{*}{ Unaffected hemisphere } & Rt. CRT & Fiber number & 1,485 & 1,718 \\
\hline & & FA & 0.441 & 0.442 \\
\hline & & $\mathrm{ADC}$ & 0.979 & 0.951 \\
\hline & Rt. CST & Fiber number & 1,242 & 1,343 \\
\hline & & FA & 0.517 & 0.519 \\
\hline & & $\mathrm{ADC}$ & 0.997 & 1.000 \\
\hline
\end{tabular}

DTT, diffusion tensor tractography; CRT, corticoreticulospinal tract; CST, corticospinal tract; FA, fractional anisotropy; ADC, apparent diffusion coefficient. 